\title{
Muscle regulatory factor gene: zebrafish (Danio rerio) myogenin cDNA
}

\author{
Yau-Hung Chen, Wen-Chih Lee, Chun-Ho Cheng, Huai-Jen Tsai* \\ Institute of Fisheries Science, National Taiwan University, 1 Roosevelt Road, Section 4, Taipei 10746, Taiwan, ROC
}

Received 24 January 2000; received in revised form 15 May 2000; accepted 24 May 2000

\begin{abstract}
Myogenin is one of the basic helix-loop-helix proteins that regulate muscle-specific gene expression. Using reverse transciption-polymerase chain reaction (RT-PCR), 5'- and 3'-rapid amplification of cDNA ends (RACE), zebrafish myogenin cDNA was cloned from mRNA of embryos at 10-96 h post-fertilization. The cDNA, at 1384 base pairs (bp), contained a 771-bp open reading frame with 113- and 500-bp flanking regions at the 5'- and $3^{\prime}$-ends, respectively. The deduced amino acid sequences of zebrafish myogenin encoded a 256-amino-acid polypeptide. In a comparison with myogenin of carp, trout, Xenopus, chicken and human, zebrafish myogenin shared 90.9, 77.6, 70.3, 62.9 and 51.5\% amino acid identity, respectively. The basic helix-loop-helix domains in myogenin are all conserved. The molecular phylogenic tree demonstrated that myogenin of zebrafish is more closely related to that of fish than to the myogenin of other vertebrates. (c) 2000 Elsevier Science Inc. All rights reserved.
\end{abstract}

Keywords: Fish; Helix-loop-helix; Molecular structure; Muscle-specific; Myogenin; Phylogenic tree; RACE; RT-PCR

\section{Introduction}

Skeletal muscle development has become a paradigm for understanding the mechanisms that control cell specification and differentiation during embryogenesis. The formation of skeletal muscle during myogenesis is controlled by four basic helix-loop-helix (bHLH) transcription factors, including MyoD (Davis et al., 1987), myogenin (Braun et al., 1989a; Edmondson and Olson, 1989; Wright et al., 1989), Myf-5 (Braun et al., 1989b) and MRF4/herculin/Myf-6 (Rhodes and Konieczny, 1989; Braun et al., 1990; Miner and Wold, 1990). The formation of specific transcription factor complexes, composed of myo-

\footnotetext{
* Corresponding author. Tel.: +886-2-23661540; fax: + 886-2-23638483.

E-mail address: hjtsai@ccms.ntu.edu.tw (H.-J. Tsai).
}

genic and ubiquitous (E12 or E47) bHLH proteins (Lassar et al., 1991), confers DNA-binding ability and facilitates the transactivation of genes containing E-boxes (CANNTG), such as the muscle creatine kinase (Jaynes et al., 1988) and myosin light chain (Braun and Arnoid, 1991).

In vertebrates, muscle fibers in the trunk and limbs are formed from myogenic cells that originate in somites (Chevallier et al., 1977; Christ et al., 1977). Somites form as epithelial balls, but soon reorganize into three regions termed dermatome, myotome, and sclerotome. Like most other vertebrates, somites of zebrafish (Danio rerio) form as epithelial spheres from the presomitic mesoderm in an anterior to posterior direction. Starting at $10 \mathrm{~h}$ post-fertilization (hpf), one pair of somites is formed every $20-30$ min by formation of a new somitic furrow (van Eeden et al., 1996). Embryonic expression in skeletal muscle of 
zebrafish is easily observable, due to the transparent nature of embryos and their rapid development. For these reasons, zebrafish has become an excellent animal model for studying myogenesis.

Previous reports have characterized myogenin cDNA in some vertebrates, such as human (Braun et al., 1989a), mouse (Edmondson and Olson, 1989), pig (Briley et al., 1995), chicken (Malik et al., 1995), Xenopus (Jennings, 1992), trout (Rescan et al., 1995) and carp (Kobiyama et al., 1998). Weinberg et al. (1996) reported that they performed in situ hybridization using a zebrafish myogenin-truncated probe and demonstrated the spatial distribution of presumptive zebrafish myogenin transcripts (Weinberg et al., 1996). However, full-length zebrafish myogenin cDNA has not been described. In this communication, we report the complete primary molecular structure of zebrafish myogenin cDNA and make comparisons with myogenins known from other vertebrate species. This information should facilitate both the understanding of myogenesis in zebrafish and the evolution of the myogenin protein.

\section{Materials and methods}

\subsection{Experimental fish}

Zebrafish of the AB strain were kept under a 14-h light and 10-h dark photoperiod at approximately $28.5^{\circ} \mathrm{C}$. Cleavage numbers and somite formation of embryos were observed with light microscopy to determine the developmental stages based on hpf (Haffer et al., 1996).

\section{2. $R N A$ isolation}

Embryos from 10 to $96 \mathrm{hpf}$ were pooled and flash-frozen in liquid nitrogen. Embryos were homogenized with TRIzol reagent (Gibco BRL), and RNA extracted according to the manufacturer's instructions.

\subsection{Reverse transcription-polymerase chain reaction $(R T-P C R)$}

First-strand cDNA was synthesized using the SuperScript Preamplification System (Gibco BRL). Degenerated oligonucleotide primers were designed with reference to polynucleotide sequences of myogenin genes from other vertebrates. A forward primer, Myog1F (ATGGAG$\mathrm{CT}(\mathrm{C} / \mathrm{G} / \mathrm{T}) \mathrm{T}(\mathrm{A} / \mathrm{T}) \mathrm{TGA}(\mathrm{A} / \mathrm{G}) \mathrm{AC}(\mathrm{A} / \mathrm{C}) \mathrm{A}(\mathrm{A} / \mathrm{C} / \mathrm{G})$ CCCCTA), and a reverse primer, Myog292R (GATGCT(C/G)TCCAC(A/G)ATAAG(A/C/G) $\mathrm{G}(\mathrm{A} / \mathrm{T})(\mathrm{A} / \mathrm{C} / \mathrm{G}) \mathrm{AG}(\mathrm{A} / \mathrm{C} / \mathrm{G}) \mathrm{GA})$, were synthesized. Thirty cycles of PCR amplification were performed using Taq DNA polymerase (Viogene). Each cycle consisted of denaturation for $40 \mathrm{~s}$ at $94^{\circ} \mathrm{C}, 1 \mathrm{~min}$ of annealing at $55^{\circ} \mathrm{C}$, and $1 \mathrm{~min}$ of extension at $72^{\circ} \mathrm{C}$. The last extension step was extended for $10 \mathrm{~min}$ at $72^{\circ} \mathrm{C}$. Amplified DNA fragments were ligated with pGEM T-Easy vector (Promega) and transformed into Escherichia coli strain DH5 $\alpha$. DNA sequencing of both the strands was carried out by using a Bigdye-Terminator Cycle Sequencing Ready reaction kit (Perkin Elmer Applied Biosystems) with a DNA sequencer (Model 310; Perkin Elmer Applied Biosystems).

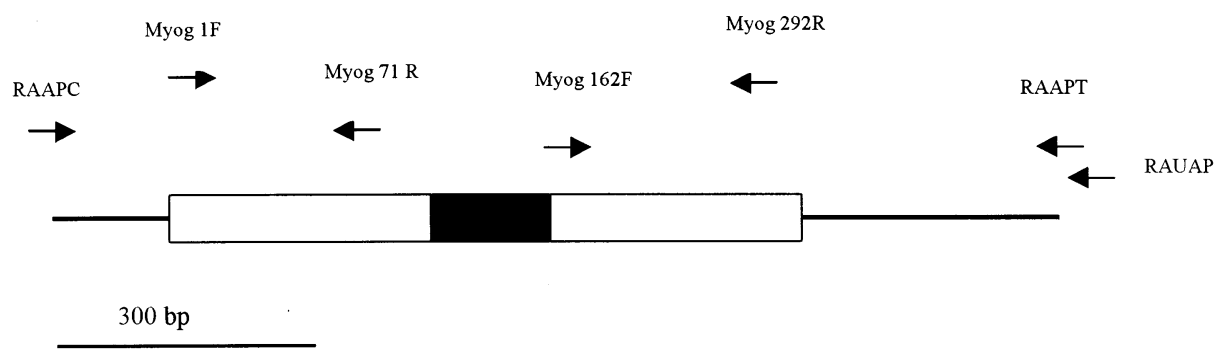

Fig. 1. The strategy of cloning cDNA encoding a fragment of zebrafish myogenin. Primers used for PCR and RACE amplification are indicated. The lines, empty boxes and closed box represent the non-coding regions, coding regions and basic helix-loop-helix domain, respectively. 
TGCAGTTGTCGTCCACAGATCTTCTTCAGAAACA 34 CCCACAAACGCTCACAAGGACCTGTACAGCTTTTCAGAGGAGACTCAGCACAGCGGGTGGACTCTAAACCAGCAAAGAC 113

ATG GAG CTT TTC GAG ACC AAC CCC TAC TTT TTC AAC GAC CAG CGT TTT TAC GAA GGC GCC 173

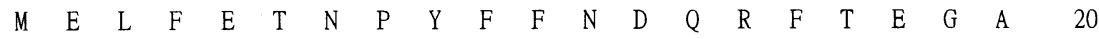

GAT AAT TTC TTC CAG TCC AGA ATC AAT GGT GGC TTC GAG CAA GCC GGA TAT CAG GAC AGG 233 $\begin{array}{lllllllllllllllllllll}D & N & F & F & Q & \text { S } & \text { R } & \text { I } & N & \text { G } & \text { G } & \text { F } & \text { E } & \text { Q } & \text { A } & \text { G } & \text { Y } & \text { Q } & \text { D } & \text { R } & 40\end{array}$

AAC TCC ATG ATG GGC TTG TGT GGG GAT GGA CGG ATG CTG ACC ACC ACA GTT GGG TTG GAA 293 $\begin{array}{lllllllllllllllllllll}\mathrm{N} & \mathrm{S} & \mathrm{M} & \mathrm{M} & \mathrm{G} & \mathrm{L} & \mathrm{C} & \mathrm{G} & \mathrm{D} & \mathrm{G} & \mathrm{R} & \mathrm{M} & \mathrm{L} & \mathrm{T} & \mathrm{T} & \mathrm{T} & \mathrm{V} & \mathrm{G} & \mathrm{L} & \mathrm{E} & 60\end{array}$

GAC AAA CCA TCT CCA TCG TCC AGC CTC GGT TTG TCC ATG TCT CCT CAT CAG GAG CAA CAG 353 $\begin{array}{llllllllllllllllllllll}\text { D } & \text { K } & \text { P } & \text { S } & \text { P } & \text { S } & \text { S } & \text { S } & \text { L } & \text { G } & \text { L } & \text { S } & \text { M } & \text { S } & \text { P } & \text { H } & \text { Q } & \text { E } & \text { Q } & \text { Q } & & 80\end{array}$

CAC TGC CCC GGC CAG TGT CTC CCC TGG GCC TGC AAG GTG TGC AAG CGC AAG TCA GTG ACT 413

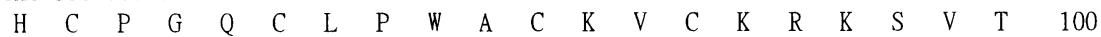

ATG GAT CGA AGA AAG GCC GCT ACC TTG AGA GAG AAG AGG AGG TTG AAG AAG GTG AAC GAG 473 $\begin{array}{llllllllllllllllllllll}M & D & R & R & K & A & A & T & \text { L } & \text { R } & \text { E } & \text { K } & \text { R } & \text { R } & \text { L } & \text { K } & \text { K } & \text { V } & \text { N } & \text { E } & 120\end{array}$

GCC TTT GAG GCT CTG AAG AGG AGC ACA TTG ATG AAC CCC AAC CAG AGG CTG CCG AAG GTG 533 $\begin{array}{lllllllllllllllllllll}\text { A } & \text { F } & \text { E } & \text { A } & \text { L } & \text { K } & \text { R } & \text { S } & \text { T } & \text { L } & \text { M } & \text { N } & \text { P } & \text { N } & \text { Q } & \text { R } & \text { L } & \text { P } & \text { K } & \text { V } & 140\end{array}$

GAG ATC CTG CGC AGT GCT ATA CAG TAC ATC GAG AGG CTG CAG GCA CTG GTC AGT TCA CTC 593 $\begin{array}{lllllllllllllllllllll}\text { E } & \text { I } & \text { L } & \text { R } & \text { S } & \text { A } & \text { I } & \text { Q } & \text { Y } & \text { I } & \text { E } & \text { R } & \text { L } & \text { Q } & \text { A } & \text { L } & \text { V } & \text { S } & \text { S } & \text { L } & 160\end{array}$

AAC CAG CAG GAG CAT GAA CAG GGG AAT CTG CAT TAT AGA GCC ACC GCC GCT GCT CCA CAT 653

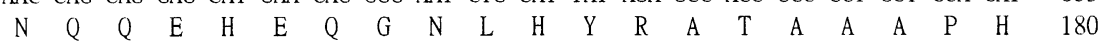

ACT GGG GTG TCG TCC TCT AGT GAT CAG GGC TCT GGC AGC ACC TGC TGC AGC AGT CCA GAA 713

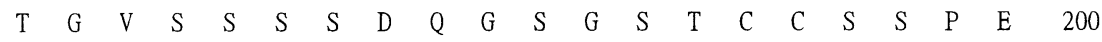

TGG AGC AGC GCG TCT GAT CAC TGT GTC CCC GCC TAT AGC TCC GCC CAC GAG GAT CTG CTG 773 $\begin{array}{llllllllllllllllllllll}\text { W } & S & S & A & S & D & H & C & \text { V } & \text { P } & \text { A } & \text { Y } & \text { S } & \text { S } & \text { A } & \text { H } & \text { E } & \text { D } & \text { L } & \text { L } & 220\end{array}$

AAC GAC GAC TCG TCA GAG CAA TCC AAC CTG AGG TCT CTG ACG TCT ATA GTG GAC AGC ATA 833 $\begin{array}{lllllllllllllllllllll}\mathrm{N} & \mathrm{D} & \mathrm{D} & \mathrm{S} & \mathrm{S} & \mathrm{E} & \mathrm{Q} & \mathrm{S} & \mathrm{N} & \mathrm{L} & \mathrm{R} & \mathrm{S} & \mathrm{L} & \mathrm{T} & \mathrm{S} & \mathrm{I} & \mathrm{V} & \mathrm{D} & \mathrm{S} & \mathrm{I} & 240\end{array}$

ACG GGA ACA GAG GCA ACT CCA GTG GCC TAT TCA GTG GAC ATT AGC AAA TAA ATCAGAAGGCC 895 $\begin{array}{lllllllllllllllllll}\text { T } & G & \text { T } & \text { E } & \text { A } & \text { T } & \text { P } & \text { V } & \text { A } & \text { Y } & \text { S } & \text { V } & \text { D } & \text { I } & \text { S } & \text { K } & * & & \end{array}$

GACTCACCGTTACCTTCAGACCAGCTTTCACTGACCACAACAGCAACATTAGACAAAGCTTTTGCAAGTTAGACAAGCC 974 TTGGAGGGCTTAATTTCCAGACAAATCTCGAGGTCCATTTGATTATTTCCAAATGTTTCTTATCTCTGTTGGAAATGAGA 1053 ATGAATTTCAGATCCCCCCTGCTGAAACTTAAGGCAGTCAGGAAGGCTGATTCATATTTTCATAGTTTGATTCCTTTAT 1132 TTTTGCATTCTGATTTTGTGTGTGCTTTATGCTTACCAATGACAAAATATTTGTGGGTCCAATGCTTTTGACCAATTTT 1211 ATTTTAATTTAAACTAGTTCCGAATGCCAGTCTTGTAAATATGTTCCTATATTTTACTACAGTGTGCTTTTTGTTATGT 1290 TATTTTGTATTTTTCCCTGTGTGTTTGCTATTATTTATTTGGACTTTTATAATAAAGATTGTTGTGTATTTGTAAAAA 1369 AAAAAAAAAAAAAAA

1384

Fig. 2. Nucleotide and deduced amino acid sequences of zebrafish (Danio rerio) myogenin cDNA. The nucleotides were numbered beginning with the first nucleotide at the $5^{\prime}$ end. Numbers on the second line of each row indicate the amino acid sequences. The polyadenylation signal (AATAAA) is underlined; the stop codon is marked with an asterisk (GenBank database accession number, AF202639).

\subsection{Rapid amplification of $c D N A$ ends (RACE)}

First-strand cDNA used for 5'-RACE was synthesized as described above, then homotailed at the $5^{\prime}$-end using terminal transferase TdT (Boehringer Mannheim) and dGTP. The resulting cDNA was then used to generate double-strand cDNA by PCR amplification in the presence of a forward primer, RAAPC (GGCCACGCGTCGACTAGTACT $\left.(\mathrm{C})_{9}\right)$, and a reverse primer, Myog71R (CAAACCGAGGCTGGACGATGGAGA). Conditions of PCR amplification were as described above with the exception of annealing at $58^{\circ} \mathrm{C}$. Basically, the procedure for $3^{\prime}$-RACE was the same as that for $5^{\prime}$-RACE, except that (1) first-strand cDNA was synthesized by a reverse 
primer, RAAPT (GGCCACGCGTCGACTAG$\left.\operatorname{TAC}(\mathrm{T})_{18}\right)$; (2) a forward primer, Myog1F, and a reverse primer, RAUAP (GGCCACGCGTCGACTAGTAC), were used to generate double-strand cDNA, and the annealing temperature was $50^{\circ} \mathrm{C}$; and (3) $1 / 50$ volume of first PCR product was used as a template for nested PCR amplification in the presence of a forward primer, Myog162F (CAGCAGGAGCATGAACAGGGGAAT), and a reverse primer, RAUAP (GGCCACGCGTCGACTAGTAC), and the annealing temperature was at $56^{\circ} \mathrm{C}$. Amplified DNA fragments were subcloned and sequenced as described above.

\subsection{Northern blot analysis}

Embryos from 21 to $24 \mathrm{hpf}$ were pooled, and their total RNAs were extracted and electrophoresed in a $1.4 \%(\mathrm{w} / \mathrm{v})$ agarose gel containing $17.5 \%$ formaldehyde. After electrophoresis, the RNAs were transferred onto a Hybond- $\mathrm{N}^{+}$ membrane (Amersham) using capillary transfer

(A)

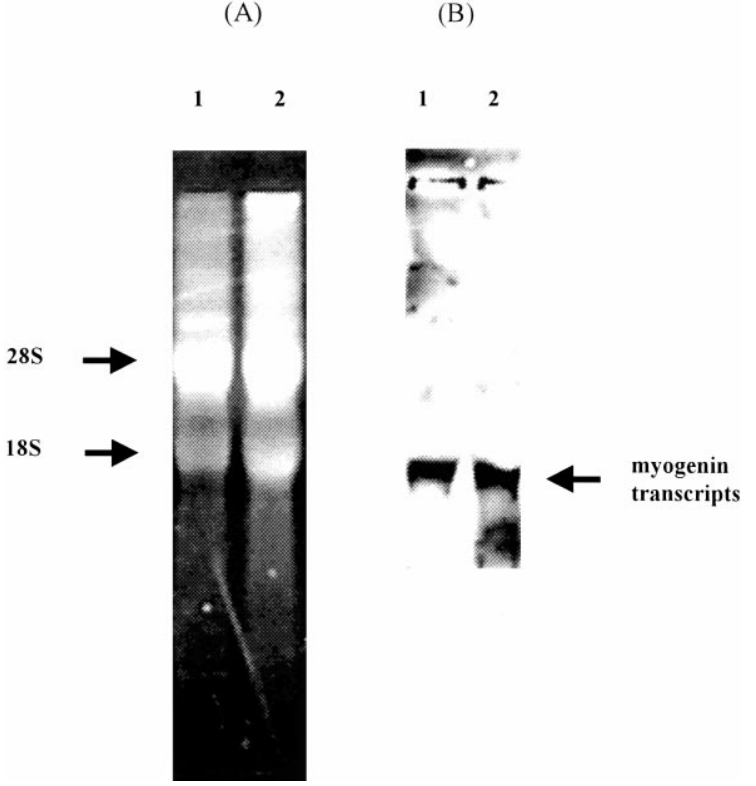

Fig. 3. Northern blot analysis of zebrafish myogenin gene transcripts. Total RNA was extracted from $21 \mathrm{hpf}$ (lane 1) and $24 \mathrm{hpf}$ (lane 2), respectively. (A) Ethidium bromide staining on a $1.4 \%(\mathrm{w} / \mathrm{v})$ agarose gel containing $17.5 \%$ formaldehyde. Arrows indicate the positions of $28 \mathrm{~S}$ and 18S rRNA. (B) Northern blot analysis using a specific probe for zebrafish myogenin gene. The arrow indicates zebrafish myogenin gene transcripts which are located approximately at position $1.4 \mathrm{~kb}$.
(Sambrook et al., 1989), and then cross-linked by UV. The DNA fragment corresponding to nucleotides 114-833 of zebrafish myogenin cDNA was labeled with digoxigenin (DIG) using a DIG RNA labeling kit (Boehringer Mannheim) and used as a probe. Hybridization was carried out in a high-SDS buffer at $70^{\circ} \mathrm{C}$ for $16 \mathrm{~h}$ by following the manufacturer's recommendations (Boehringer Mannheim). Washing conditions were $0.5 \times \mathrm{SSC}$ at $70^{\circ} \mathrm{C}$ for $30 \mathrm{~min}$, followed by $0.1 \times \mathrm{SSC}$. CDP-STAR (Tropix) was used as a substrate to visualize the positive bands after autoradiography for $20 \mathrm{~min}$.

\subsection{Analyses of polypeptide structure and phylogenic dendrogram}

The presumptive amino acid sequence was determined with the Wisconsin Sequence Analysis Package version 10.0 (GCG), provided by the National Health Research Institute of the Republic of China. The Gap program was used for pairwise comparison. Pileup and Prettybox programs were used for multiple comparisons. The molecular evolution genetic analysis (MEGA) program and neighbor-joining methods were used for phylogenic tree analysis.

\section{Results and discussion}

\subsection{Nucleotide and deduced amino acid sequences of zebrafish myogenin cDNA}

Primers designed for RT-PCR and RACE and used to clone full-length zebrafish myogenin cDNA are illustrated in Fig. 1. A 720-bp fragment was amplified by the primers, Myog1F and Myog292R. A 326-bp fragment was amplified by using the primers, RAAPC and Myog71R, which corresponded to the 5 -end non-coding region. For 3'-RACE, we used the primers, Myog67F and RAAPT, for the first PCR, then used the primers, Myog162F and RAUAP, for the second PCR, resulting in a 788-bp fragment. The full-length cDNA of zebrafish myogenin was 1384 bp containing a 771-bp open reading frame with 113and 500-bp flanking region at the $5^{\prime}$ - and $3^{\prime}$-ends, respectively (Fig. 2). The deduced amino acid sequence of zebrafish myogenin revealed a 256amino-acid polypeptide. 


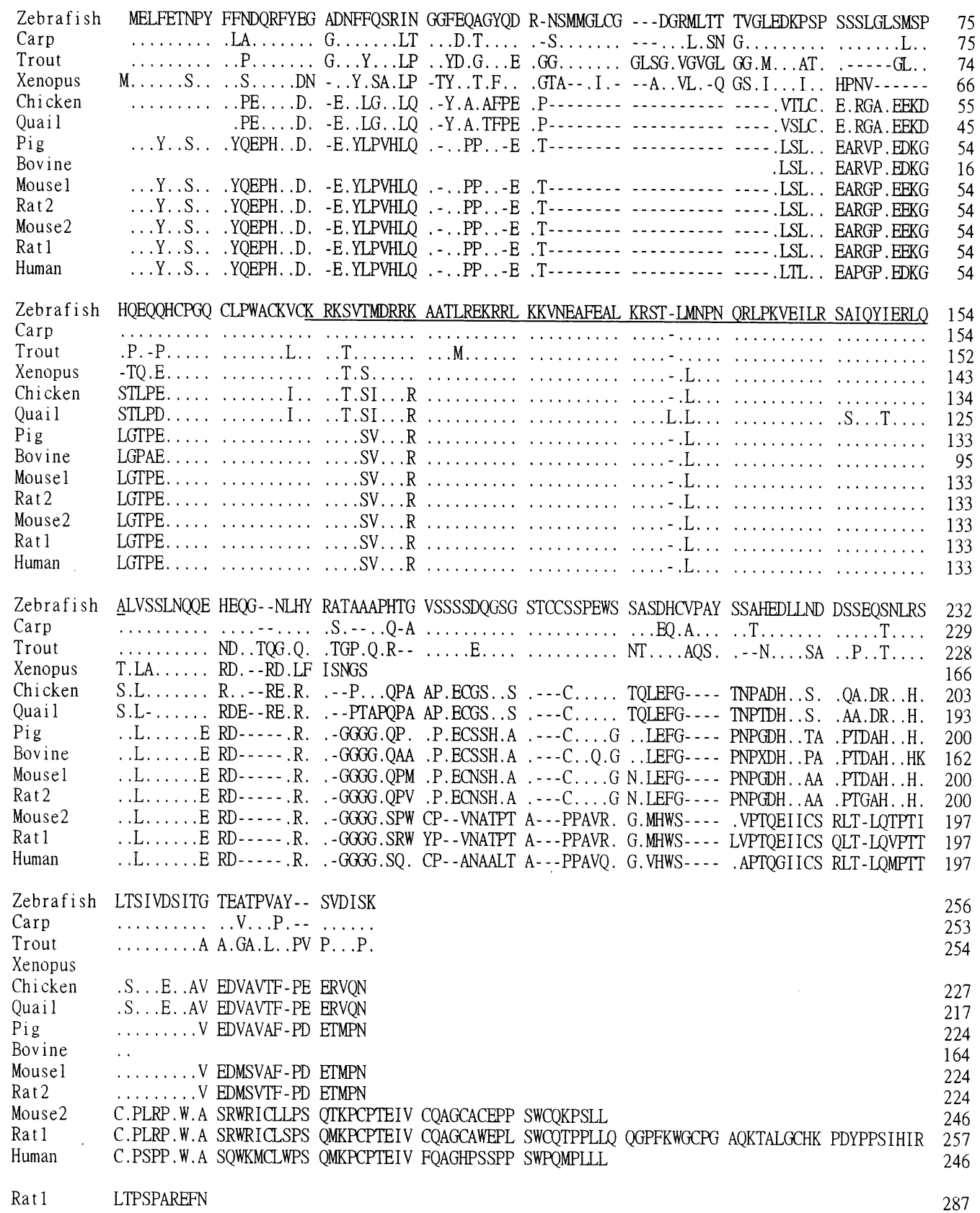

Fig. 4. Comparison of the deduced amino acid sequence of zebrafish myogenin with those of other known vertebrates. Data were obtained from GenBank nucleotide sequence database with the following accession numbers, carp (AB012881), trout (Z46912), Xenopus (S34390), chicken (D90157), quail (L15473), pig (X89007), bovine (AF091714), mouse 1 (D90156), rat 2 (AF054894), mouse 2 (X15784), rat 1 (M24393) and human (X17651) myogenin. Amino acid residues identical to that of zebrafish myogenin are represented by dots. The underlining indicates the basic helix-loop-helix domain. Dashes represent gaps created to maximize the degree of identity among all compared sequences.

\subsection{Northern blot analysis}

In order to confirm the full length of the zebrafish myogenin transcript, we performed Northern blot analysis. Total RNAs were extracted from embryos at 21 and $24 \mathrm{hpf}$ and used to generate a Northern blot which was then hybridized with a DIG-labeled probe corresponding to nucleotide positions from 114 to 833 of zebrafish myogenin cDNA. A positive signal with a 
molecular mass of around $1.4 \mathrm{~kb}$ was detected (Fig. 3), suggesting that the actual full-length of zebrafish myogenin cDNA was $1.4 \mathrm{~kb}$. These data are consistent with the results obtained from RTPCR and RACE.

\subsection{Comparison with known myogenin of other vertebrates}

Although both the 5'- and 3'-untranslated regions of the zebrafish myogenin cDNA differ substantially among vertebrates, the deduced amino acid sequence of zebrafish myogenin shared 90.9, 77.6, 70.3, 62.9 and 51.5\% amino acid identity with carp (Kobiyama et al., 1998), trout (Rescan et al., 1995), Xenopus (Jennings, 1992), chicken (Malik et al., 1995) and human (Braun et al., 1989a) myogenins, respectively. When the $\mathrm{N}$ - and C-terminal amino acid sequences of myogenin were compared among carp, trout, Xenopus and other known vertebrate species, we concluded that zebrafish myogenin is more closely related to that of fish (carp and trout) than to the myogenin of other known vertebrate species.

MRF family proteins (MyoD, Myf-5, myogenin and MRF4) have a conserved bHLH domain (Lassar et al., 1991). The HLH domain can form a heterodimer with ubiquitous bHLH proteins (E12 or E47), and can bind to the E-box by the basic region. This complex regulates muscle-specific gene expression (Jaynes et al., 1988). Zebrafish myogenin also contained this characteristic bHLH region (Fig. 4). There was a significant difference in the basic regions between

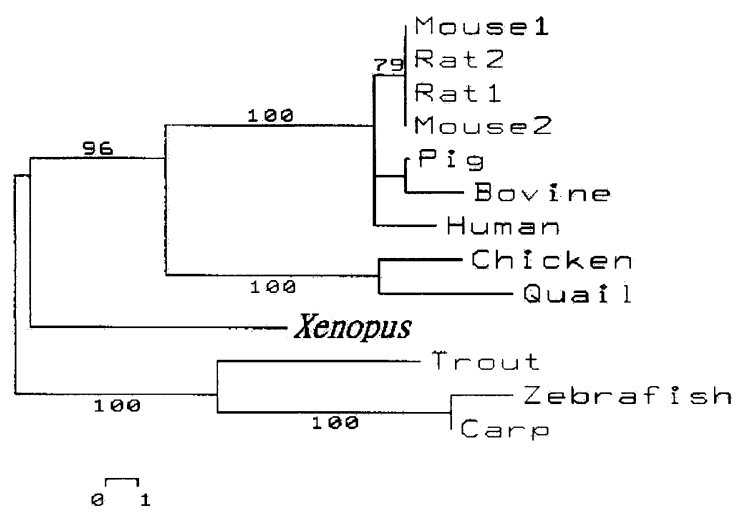

Fig. 5. A molecular phylogenic tree of myogenin polypeptides. This dendrogram is based on amino acid residues of the position from 41 to 164 of zebrafish myogenin. zebrafish myogenin (KRKSVTMDRRKAA) and MyoD (KRKTTNADRRKAA) (Weinberg et al., 1996): the amino acid residues of SVTM of myogenin versus those of TTNA of MyoD. Interestingly, the corresponding amino acid sequences of carp and Xenopus myogenins also differed from that of MyoD. It is worthwhile to note that the basic region of $\mathrm{MyoD}$ is identical among four species (zebrafish, trout, carp and Xenopus). In contrast, the basic region of myogenin is relatively more variable.

In order to determine the molecular phylogenic relationship of zebrafish myogenin with that of other known vertebrates, the MEGA program and neighbor-joining methods were used. The molecular phylogenic tree of myogenin showed that zebrafish myogenin was more closely related to that of carp and trout than to the myogenin of other species (Fig. 5). This evidence agrees with the results obtained from amino acid comparisons.

\section{Acknowledgements}

The authors wish to thank Institute of Zoology, Academia Sinica, for providing the founders of zebrafish $A B$ strain. This work was supported by the National Science Council, Republic of China.

\section{References}

Braun, T., Arnoid, H.H., 1991. The four human muscle regulatory helix-loop-helix proteins $M y f 3-M y f 6$ exhibit similar hetero-dimerization and DNA binding properties. Nucleic Acids Res. 19, 5645-5651.

Braun, T., Bober, E., Buschhausen-Denker, G., Kohtz, S., Grzeschik, K.H., Arnold, H.H., 1989a. Differential expression of myogenic determination genes in muscle cells: possible autoactivation by Myf gene products. EMBO J. 8, 3617-3625.

Braun, T., Buschhausen-Denker, G., Bober, E., Tannich, E., Arnold, H.H., 1989b. A novel human muscle factor related to but distinct from MyoD1 induces myogenic conversion in $10 \mathrm{~T} 1 / 2$ fibroblasts. EMBO J. 8, 701-709.

Braun, T., Bober, E., Winter, B., Rosenthal, N., Arnold, H.H., 1990. Myf-6, a new member of the human gene family of myogenic determination factors: evidence for a gene cluster on chromosome 12 . EMBO J. 9, 821-831. 
Briley, G.P., Reecy, J.M., Grant, A.L., Bidwell, C.A., 1995. Cloning and expression of the porcine myogenin gene. Anim. Biotechnol. 6, 79-92.

Chevallier, A., Kieny, M., Mauger, A., 1977. Limbsomite relationship: origin of the limb musculature. J. Embryol. Exp. Morphol. 41, 245-258.

Christ, B., Jacob, H.J., Jacob, M., 1977. Experimental analysis of the origin of the wing musculature in avian embryos. Anat. Embryol. (Berlin) 150, $171-$ 186.

Davis, L.R., Weintraub, H., Lassar, A.B., 1987. Expression of a single transfected cDNA converts fibroblasts to myoblasts. Cell 51, 987-1000.

Edmondson, D.G., Olson, E.N., 1989. A gene with homology to the $m y c$ similarity region of $M y o D 1$ is expressed during myogenesis and is sufficient to activate the muscle differentiation program. Genes Dev. 3, 628-640.

Haffer, P., Granato, M., Brand, M., Mullins, M.C., Hammerschmidt, M., Kane, D.A., Odenthal, J., van Eeden, F.J.M., Jiang, Y.J., Heisenberg, C.P., Kelsh, R.N., Furutani-Seiki, M., Vogelsang, E., Beuchle, D., Schach, U., Fabian, C., Nusslein-Volhard, C., 1996. The identification of genes with unique and essential functions in the development of the zebrafish, Danio rerio. Development 123, 1-36.

Jaynes, J.B., Johnson, J.E., Buskin, J.N., Hauschka, S.D., 1988. The muscle creatine kinase gene is regulated by multiple upstream elements, including a muscle-specific enhancer. Mol. Cell. Biol. 8, 62-70.

Jennings, C.G., 1992. Expression of the myogenic gene MRF4 during Xenopus development. Dev. Biol. 150, 121-132.

Kobiyama, A., Nihei, Y., Hirayama, Y., Kikuchi, K., Suetake, H., Johnston, I.A., Watabe, S., 1998. Molecular cloning and developmental expression patterns of the MyoD and MEF2 families of muscle transcription factors in the carp. J. Exp. Biol. 201, 2801-2813.

Lassar, A.B., Davis, R.L., Wright, W.E., Kadesch, T., Murre, C., Voronova, A., Baltimore, D.,
Weintraub, H., 1991. Functional activity of myogenic HLH proteins requires hetero-oligomerization with E12/E47-like proteins in vivo. Cell 66, 305315.

Malik, S., Huang, C.F., Schmidt, J., 1995. The role of the CANNTG promoter element (E box) and the myocyte-enhancer-binding-factor-2 (MEF-2) site in the transcriptional regulation of the chick myogenin gene. Eur. J. Biochem. 230, 88-96.

Miner, J.H., Wold, B., 1990. Herculin, a fourth member of the MyoD family of myogenic regulatory genes. Proc. Natl. Acad. Sci. USA 87, 1089-1093.

Rescan, P.Y., Gauvry, L., Paboeuf, G., 1995. A gene with homology to myogenin is expressed in developing myotomal musculature of the rainbow trout and in vitro during the conversion of myosatellite cells to myotubes. J. FEBS Lett. 362, 89-92.

Rhodes, S.J., Konieczny, S.F., 1989. Identification of MRF4: a new member of the muscle regulatory factor gene family. Genes Dev. 3, 2050-2061.

Sambrook, J., Fritsch, E.F., Maniatis, T., 1989. Molecular Cloning: A Laboratory Manual. Cold Spring Harbor Laboratory Press, Cold Spring Harbor, NY.

van Eeden, F.J., Granato, M., Schach, U., Brand, M., Furutani-Seiki, M., Haffter, P., Hammerschmidt, M., Heisenberg, C.P., Jiang, Y.J., Kane, D.A., Kelsh, R.N., Mullins, M.C., Odenthal, J., Warga, R.M., Allende, M.L., Weinberg, E.S., Nüsslein-Volhard, C., 1996. Mutations affecting somite formation and patterning in the zebrafish, Danio rerio. Development 123, 153-164.

Weinberg, E.S., Allende, M.L., Kelly, C.S., Abdelhamid, A., Murakami, T., Andermann, P., Doerre, O.G., Grunwald, D.J., Riggleman, B., 1996. Developmental regulation of zebrafish MyoD in wildtype, no tail and spadetail embryos. Development 122, 2711-2780.

Wright, W.E., Sassoon, D.A., Lin, V.K., 1989. Myogenin, a factor regulating myogenesis, has a domain homologous to MyoD. Cell 56, 607-617. 